\title{
Modelling of elliptical core D-fibers for determination of cladding thickness
}

\author{
Sameer M. Chandani", Nicolas A.F. Jaeger \\ Dept. of Electrical \& Computer Engineering, University of British Columbia, Vancouver, BC, \\ Canada
}

\begin{abstract}
Cladding-reduced fibers with elliptical cores can be used in the fabrication of fiber-optic sensors. Here we analyze the power transmission ratio in cladding reduced D-shaped optical fibers for the purpose of determining the cladding thickness, in a non-destructive manner, when the fibers have been etched to reduce their cladding thicknesses to allow interaction of the optical evanescent field with an external medium having a refractive index greater than the mode effective index of the fiber. Parameters needed to determine the cladding thickness are determined empirically using the measured power transmission ratio of a few calibration fibers. We show that one can estimate the cladding thickness by fitting the measured transmission ratio curves for the fiber of interest to the curves generated for the calibration fibers.
\end{abstract}

Keywords: D-fiber, fiber modelling, optical fiber measurements, cladding thickness

\section{INTRODUCTION}

D-SHAPED optical fibers are commonly used in the fabrication of evanescent wave-based fiber-optic sensors ${ }^{1,2,3,4,5}$. Typically, the cladding thickness of such fibers is reduced by etching in hydrofluoric acid to allow access to the evanescent field. The etch rate depends on factors such as the acid concentration, temperature, humidity, composition of the cladding material and the fiber batch $^{6}$. Due to these variables, timed etches are not very accurate and a more accurate and reliable method is needed to control the etch depth.

There have been several methods proposed to determine the cladding thickness of D-fibers and/or the polish depth of side-polished fibers. For example, several liquids having various refractive indices are dropped onto a side-polished fiber and the resulting power loss of the fiber for each liquid is fit to a model to determine the polish depth ${ }^{7}$. An in-situ method is presented whereby the birefringence of a polarization-maintaining D-fiber is monitored while being etched ${ }^{8}$. This method is limited to cladding thicknesses of about $1.5 \mu \mathrm{m}$ or less. We have previously demonstrated a nondestructive method to determine the cladding thickness of a step-index D-shaped optical fiber using a thermo-optic oil and three temperature measurements ${ }^{9}$. The measured power transmission ratio of the D-fiber immersed in a thermo-optic oil is fit to a theoretical model that is based on a method proposed by Sharma et al. ${ }^{10}$.

In this paper we make an additional modification to Sharma's method to account for a particular D-fiber's actual refractive index distribution. We use an empirical parameter in the model obtained by fitting two measured responses for the particular fiber to the model.

\section{DESCRIPTION OF THEORETICAL MODEL}

Fig. 1 shows the cross section (not to scale) of the D-fiber used in our work (obtained from KVH Industries). The fiber has a nominally elliptical core of semimajor axis $a$ and semiminor axis $b$ with its major axis parallel to the fiber flat. The core is approximately $4 \mu \mathrm{m}$ wide and $2 \mu \mathrm{m}$ thick with its center located approximately $14 \mu \mathrm{m}$ below the fiber flat.

*sameerc@ece.ubc.ca; phone 1-604-822-6268; fax 1-604-822-5949

Photonics North 2007, edited by John Armitage,

Proc. of SPIE Vol. 6796, 67963M, (2007)

0277-786X/07/\$18 - doi: 10.1117/12.778948

Proc. of SPIE Vol. 6796 67963M-1 
The core has a graded index and is surrounded by a fluorine-doped cladding. There exists a small undoped silica circular region in the center of the core, known as a vapour spot, which is an artifact of the fabrication process ${ }^{11,12}$. The exact profiles of the graded index of the core and of the vapor spot are not known. The cladding thickness, $d$, is defined as the minimum distance between the fiber core edge and the fiber flat. The fiber is polarization-maintaining.

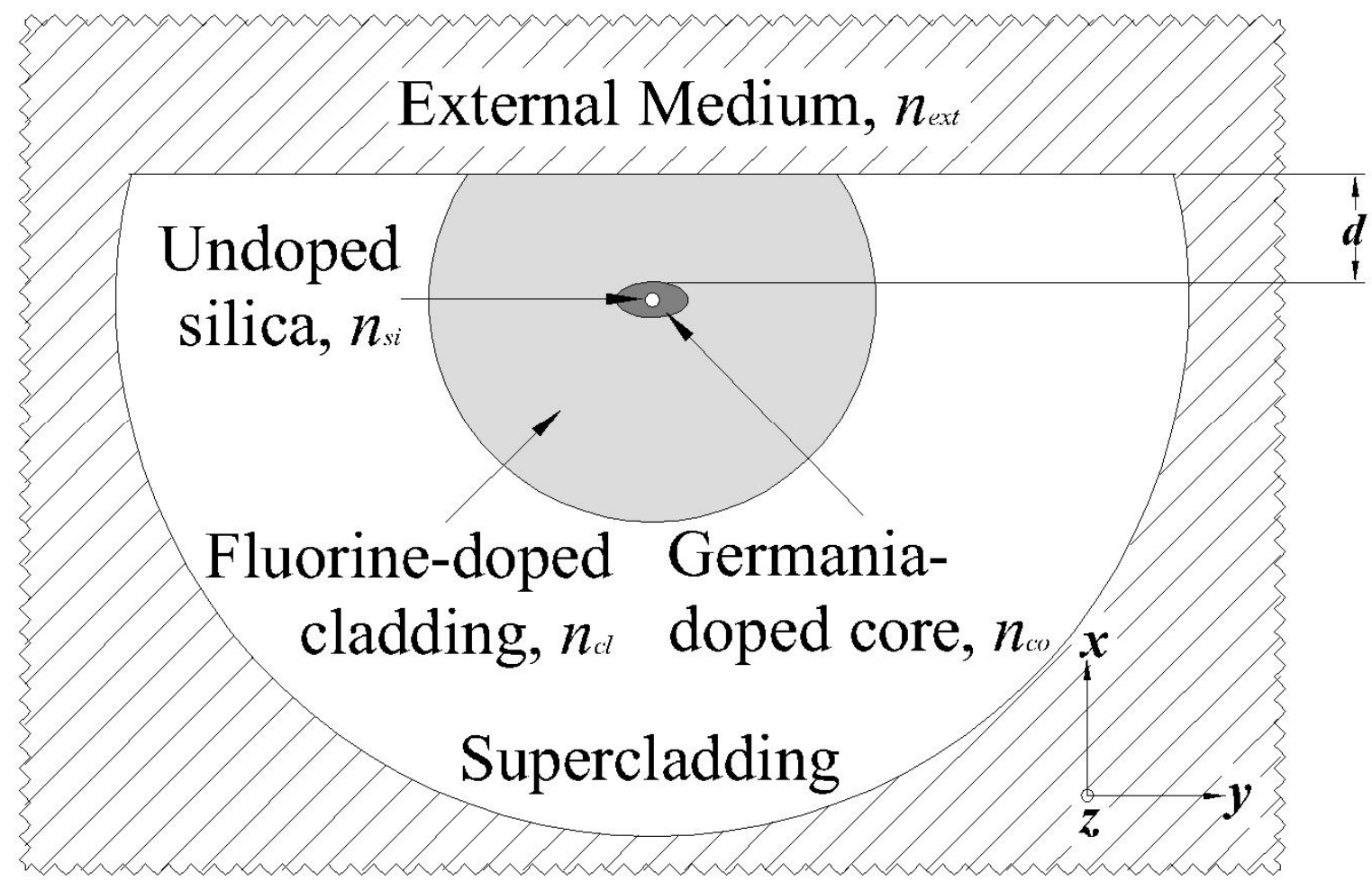

Fig. 1. Cross sectional diagram of D-fiber immersed in an external medium. Typical specifications: $n_{c o}=1.4756, n_{c l}=1.441, n_{s i}=$ $1.444, a=2 \mu \mathrm{m}, b=1 \mu \mathrm{m}$ at $\lambda_{\mathrm{o}}=1550 \mathrm{~nm}$.

In a typical optical fiber, the cladding refractive index $n_{c l}$ is less than the core refractive index $n_{c o}$ and the mode effective index $n_{\text {eff }}$ lies between $n_{c l}$ and $n_{c o}$. In the case of a D-fiber immersed in an external medium, having a refractive index $n_{\text {ext }}$, the fiber mode becomes leaky when $n_{\text {ext }}>n_{\text {eff: }}$. To model the fiber immersed in an external medium, we first replace the elliptical core with an equivalent planar waveguide, as described by Sharma et al. This equivalent planar waveguide is then extended to a four-layer planar waveguide to take into account the external medium, which acts as a semiinfinite superstrate. For the case where $n_{\text {ext }}>n_{\text {eff }}$, the mode becomes leaky and its propagation constant is complex with real and imaginary parts $\beta_{r}$ and $\beta_{i}$, respectively. The $\mathrm{TE}_{0}$ mode's propagation constant for the four-layer waveguide is given analytically by Thyagarajan et al. ${ }^{13}$ The power transmission ratio, $T_{P}$, can then be written in terms of the power into, $P_{\text {in }}$, and the power out of, $P_{\text {out }}$, a section of fiber of length $L$, as

$$
T_{P}=\frac{P_{o u t}}{P_{\text {in }}}=e^{-2 \beta_{i} L}
$$

The empirical formulas provided by Sharma have been demonstrated for circular step-index profile fibers. The technique matches, as far as possible, the modal fields in the direction of interaction (the $x$-direction, see Fig. 1) and the propagation constants of the circular fiber and its equivalent planar waveguide. Fig. 2 shows schematically the circular core superimposed on its equivalent planar waveguide (identified by the dashed lines) along with their respective core dimensions and refractive index distributions.

Sharma's empirical formulas for $\sigma, n_{x l}$ and $n_{x 2}$ are repeated here for convenience ${ }^{10}$ 


$$
n^{2}(x)= \begin{cases}n_{x 1}^{2}=n_{c o}^{2}-\left(U^{2}-p^{2}\right) /\left(k_{o} b\right)^{2}, & |x|<b \sigma \\ n_{x 2}^{2}=n_{x 1}^{2}-p^{2} \sec ^{2}(p \sigma) /\left(k_{o} b\right)^{2}, & |x|>b \sigma\end{cases}
$$

with

$$
\begin{gathered}
p^{3}=1.3528+1.6880 \mathrm{~V}-0.1894 V^{2} \\
\sigma=0.8404+0.0251 V-0.0046 V^{2}
\end{gathered}
$$

where $U=b \sqrt{k_{o}^{2} n_{c o}^{2}-\beta_{o}^{2}}, V=k_{o} b \sqrt{n_{c o}^{2}-n_{c l}^{2}}, \beta_{o}$ and $k_{o}$ are the fiber mode and free space propagation constants, respectively.

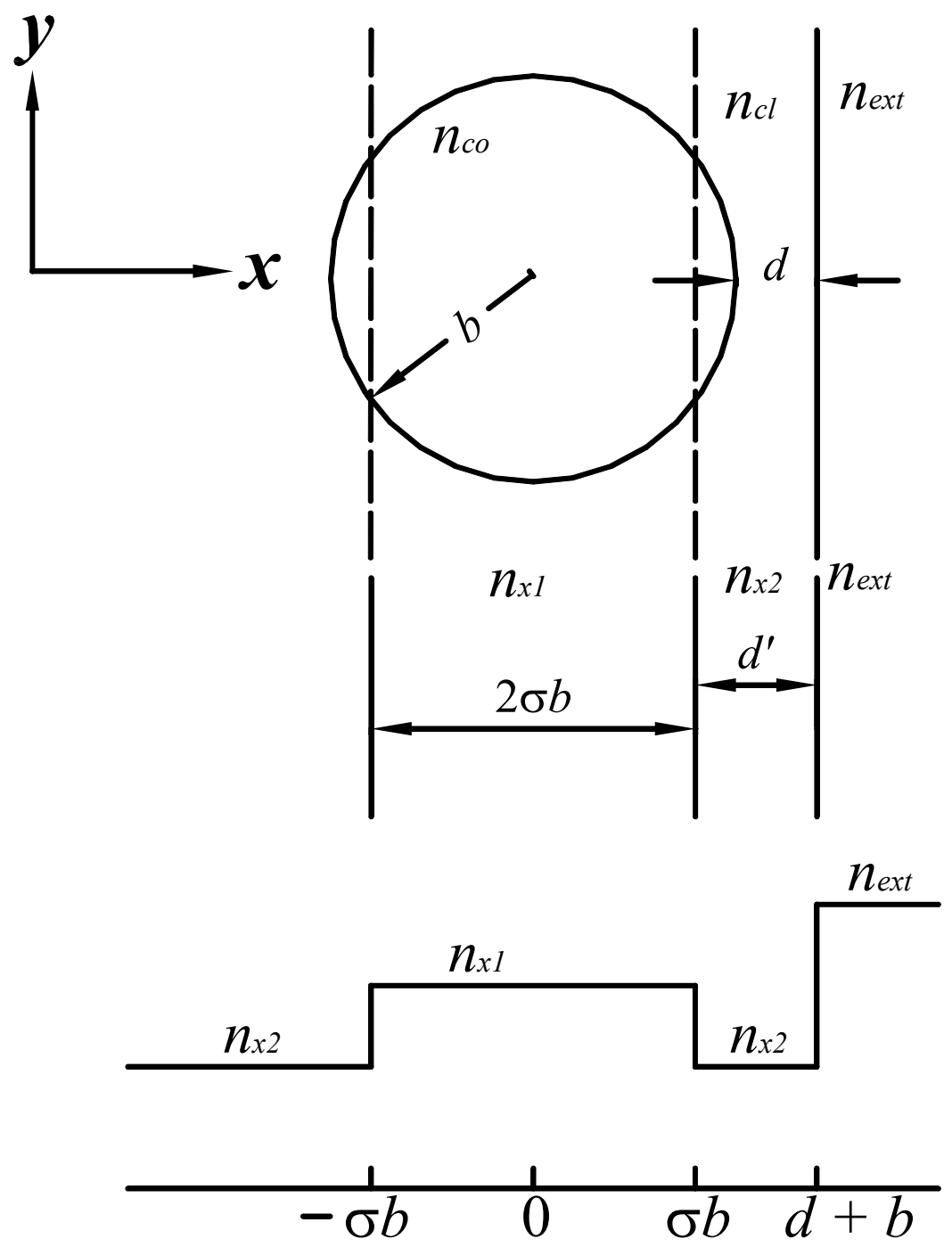

Fig. 2. Schematic of circular core and its equivalent planar waveguide with cladding thickness $d$ and an external medium of refractive index $n_{\text {ext }}$. Circular core of radius $b$, core and cladding refractive indices of $n_{c o}$ and $n_{c l}$. Equivalent planar waveguide (identified by the dashed lines) of thickness $2 \sigma b$, core and cladding refractive indices of $n_{x l}$ and $n_{x 2}$.

Here, to model our D-fiber immersed in an external medium, we start by applying Sharma's technique to our D-fiber, assuming a step-index profile. The resulting equivalent planar waveguide has a propagation constant $\beta_{E P W}$ and a modal 
field $\Psi_{E P W}(x)$. To verify Sharma's technique, the commercially available waveguide modelling software BeamPROPTM was used to model the step-index D-fiber. The propagation constant determined using BeamPROPTM was virtually identical to $\beta_{E P W}$, verifying that Sharma's model matches the propagation constant well for an elliptical step-index profile fiber. The modal fields, however, are not well matched, requiring a modification as described below.

According to Sharma's technique, the thickness of the cladding layer of the four-layer waveguide, $d^{\prime}$, should be $d+(1-\sigma) b$ (see Fig. 2). However, to take into account the poor match between the modal fields of the actual D-fiber and its equivalent planar waveguide we propose shifting the D-fiber's equivalent planar waveguide. We propose that $d^{\prime}=d+d_{\text {shift }}$, where $d_{\text {shift }}$ is empirically determined for a particular fiber. The shift of the modal field achieves a better match between the portion of optical power that penetrates into the external medium in the model and that which penetrates into the external medium in the actual fiber. Fig. 3 shows a schematic of an elliptical core fiber and its equivalent planar waveguide (identified by the dashed lines) with a positive shift.

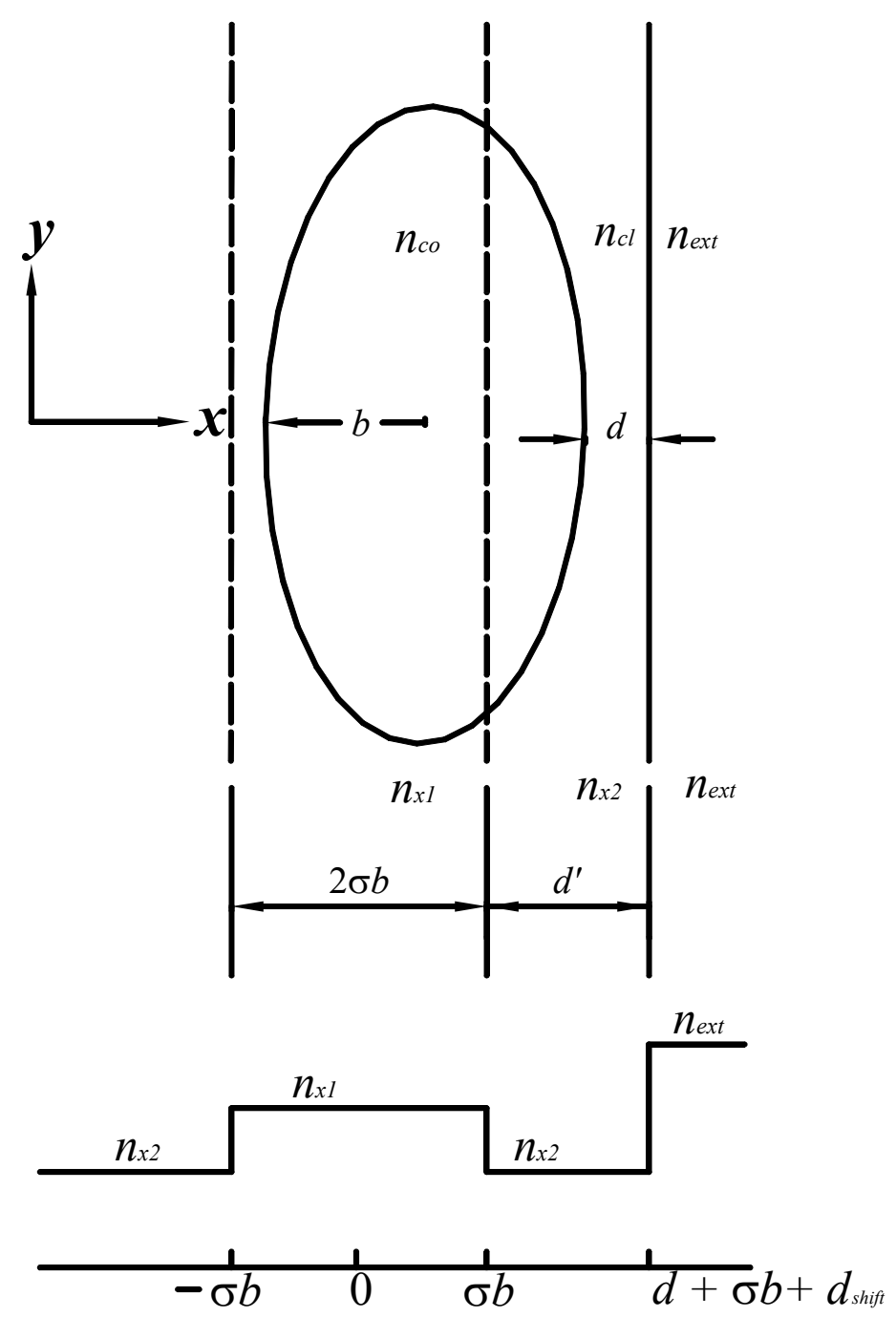

Fig. 3. Schematic of elliptical core and its equivalent planar waveguide (identified by the dashed lines), shifted by $d_{\text {shift, }}$ with cladding thickness $d$ and an external medium of refractive index $n_{\text {ext }}$. Elliptical core of semiminor axis $b$, core and cladding refractive indices of $n_{c o}$ and $n_{c l}$. Equivalent planar waveguide of thickness $2 \sigma b$, core and cladding refractive indices of $n_{x l}$ and $n_{x 2}$.

\section{DETERMINATION OF $d_{\text {shift }}$}


To determine $d_{\text {shift }}$, we used the measured transmission ratio of two sections of D-fiber, both taken from a single longer fiber, that were etched so that each section had a different cladding thickness from the other one. In particular, we used one section with a shallow etch and one with a deep etch. To demonstrate the method, we used the five fiber sections, all taken from the same fiber, that were used in our previous work ${ }^{9}$. After measuring their transmission ratios, the sections were cleaved through the centers of their etched regions and scanning electron microscope (SEM) images were used to determine their respective cladding thicknesses. The experimental technique involves the measurement of the transmission ratio, $T_{P}$ and the slope, $M_{P}$ of the transmission ratio at $25^{\circ} \mathrm{C}^{9} . T_{P}$ and $M_{P}$ are then fit to the model by using $d$ and $b$ as fitting parameters. The semiminor axis $b$ plays a role in determining the power transmission ratio and is needed as a fitting parameter to determine the cladding thickness ${ }^{9}$. The resulting $d$ and $b$ values that give the best fit, in a least squares sense, between the measured and calculated transmission ratios and their slopes are $d_{f i t}$ and $b_{f i t}$. Table 1 shows the measured transmission ratios, their slopes and the cladding thicknesses measured using the SEM images, $d_{\text {SEM }}$, for the five fiber sections used in this work.

Table 1. Measured transmission ratios, slopes and cladding thicknesses of D-fiber sections

\begin{tabular}{cccc}
\hline \hline Section & $d_{\text {SEM }}( \pm 0.2 \mu \mathrm{m})$ & $T_{P}$ & $M_{P}\left(\times 10^{-4} /{ }^{\circ} \mathrm{C}\right)$ \\
\hline S1 & 6.3 & 0.877 & -5.90 \\
S2 & 5.5 & 0.788 & -9.75 \\
S3 & 4.5 & 0.516 & -16.67 \\
S4 & 4.0 & 0.161 & -13.53 \\
S5 & 3.8 & 0.236 & -12.26 \\
\hline \hline
\end{tabular}

To start with, we fit the measured transmission ratio and slope of each section to the model and determined a $b_{f i t}$ and $d_{\text {shift }}$ value so that $d_{f i t}=d_{S E M}$ for each section. Shown in Table 2 are the model parameters required such that $d_{f i t}=d_{S E M}$ for each section.

Table 2. Model parameters required to match cladding thicknesses determined using SEM images and the present model

\begin{tabular}{cccc}
\hline \hline Section & $d_{\text {fit }}=d_{\text {SEM }}(\mu \mathrm{m})$ & $b_{\text {fit }}(\mu \mathrm{m})$ & $d_{\text {shift }}(\mu \mathrm{m})$ \\
\hline S1 & 6.3 & 0.90 & 1.1200 \\
S2 & 5.5 & 0.90 & 1.3630 \\
S3 & 4.5 & 0.91 & 1.3202 \\
S4 & 4.0 & 0.94 & 0.6798 \\
S5 & 3.8 & 1.00 & 0.6969 \\
\hline \hline
\end{tabular}

The modal fields of the D-fiber's equivalent planar waveguide depend on the semiminor axis $b$ and thus $d_{\text {shift }}$ is also a function of $b$. We use the fiber sections S1 and S5 as the calibration sections since their values for $d_{S E M}$ are at the two extremes of the five sections, where S1 is the shallow etched section and S5 is the deep etched one. We used the $d_{\text {shift }}$ and $b_{f i t}$ values for $\mathrm{S} 1$ and $\mathrm{S} 5$ to obtain a linear function that will give $d_{\text {shift }}$ for any value of $b$, given as

$$
d_{\text {shift }}=-4.2310 b+4.9279
$$

The transmission ratio and slope values of each of the five sections were fit to the model ${ }^{9}$ using (4) to calculate $d_{\text {shiff }}$. The results are shown in Table 3.

Table 3. Measured and calculated values for the cladding thickness using sections S1 and S5 as the calibration pair

\begin{tabular}{cccc}
\hline \hline \multicolumn{4}{c}{ S1 and S5 } \\
\hline \multicolumn{4}{c}{$d_{\text {shift }}=-4.2310 b+4.9279$} \\
\hline \hline Section & $d_{\text {SEM }}( \pm 0.2 \mu \mathrm{m})$ & $d_{\text {fit }}(\mu \mathrm{m})$ & $\Delta d=\mid d_{\text {SEM }}-d_{\text {fit }}(\mu \mathrm{m})$ \\
\hline S1 & 6.3 & 6.3 & 0.0 \\
S2 & 5.5 & 5.8 & 0.3 \\
S3 & 4.5 & 4.8 & 0.3 \\
S4 & 4.0 & 3.7 & 0.3 \\
S5 & 3.8 & 3.8 & 0.0 \\
\hline \hline
\end{tabular}


Since we used S1 and S5 as the calibration sections, the $\Delta d$ 's $\left(\Delta d=\left|d_{S E M}-d_{f i t}\right|\right)$ for S1 and S5 are zero and for the other three sections are $0.3 \mu \mathrm{m}$, which is an improvement over our previously reported results ${ }^{9}$, demonstrating that a calibration using one shallow and one deep etched section to determine $d_{\text {shift }}$ gives good results. Clearly there are three other possible combinations of deep and shallow etched sections that could have been used to obtain a linear function for $d_{\text {shift. }}$ However, upon inspection of Table 1, it is evident that the transmission ratio values of S4 and S5 do not fit the general trend of decreasing transmission with decreasing cladding thickness. Based on modelled transmission curves, we suspect that either the measured transmission ratio, slope or cladding thickness of S4 is incorrect and will not use it as a calibration section. Table 4 shows the results of using S2 and S5 as the shallow and deep etch calibration section, respectively.

Table 4. Measured and calculated values for the cladding thickness using sections S2-S5 the as calibration pair

\begin{tabular}{cccc}
\hline \hline \multicolumn{4}{c}{ S2 and S5 } \\
\hline \multicolumn{4}{c}{$d_{\text {shift }}=-6.661 b+7.3579$} \\
\hline \hline Section & $d_{\text {SEM }}( \pm 0.2 \mu \mathrm{m})$ & $d_{\text {fit }}(\mu \mathrm{m})$ & $\Delta d=\left|d_{\text {SEM }}-d_{\text {fit }}\right|(\mu \mathrm{m})$ \\
\hline S1 & 6.3 & 6.4 & 0.1 \\
S2 & 5.5 & 5.5 & 0.0 \\
S3 & 4.5 & 4.5 & 0.0 \\
S4 & 4.0 & 3.6 & 0.4 \\
S5 & 3.8 & 3.8 & 0.0 \\
\hline \hline
\end{tabular}

\section{CONCLUSION}

We have presented a method for determining the cladding thickness, after being reduced by etching, in elliptical core D-shaped fibers. The measured transmission ratio and slope of an etched fiber can be fit to a model to determine it's cladding thickness. An empirical parameter used in the model is first determined using two calibration sections of the fiber. We show that a shallow etch and a deep etch calibration section can be used to determine the empirical parameter, $d_{\text {shift }}$. Comparisons of the differences between the cladding thicknesses as measured using SEM images and those determined using the present method illustrate the effectiveness of the method presented.

\section{ACKNOWLEDGMENTS}

This research has been supported in part by the National Sciences and Engineering Research Council of Canada.

\section{REFERENCES}

1. X. Chen, K. Zhou, L. Zhang, I Bennion, "Optical chemsensors utilizing long-period fiber gratings UV-inscribed in D-fiber with enhanced sensitivity through cladding etching," IEEE Photonics Tech. Lett., vol. 16, pp. 1352-4, 2004.

2. W. Jin et al., "Compensation for surface contamination in a D-fiber evanescent wave methane sensor," IEEE J. Lightwave Tech., vol. 13, pp. 1177-83, 1995.

3. S.M. Chandani, N.A.F. Jaeger, "Fiber-optic temperature sensor using evanescent fields in D-fibers," IEEE Photonics Tech. Lett., vol. 17, pp. 2706-08, 2005.

4. M-.H Chiu, S-.F. Wang and R-.S. Chang, "D-type fiber biosensor based on surface-plasmon resonance technology and heterodyne interferometry," Optics Letters, vol. 30, no. 3, pp. 233-235, Feb. 2005.

5. K.H. Smith et al., "Surface-relief fiber Bragg gratings for sensing applications," Applied Optics, vol. 45, no. 8, pp. 1669-75, March 2006.

6. X. Chen, B. Yu, Y. Zhu, A. Wang, "Deep wet etching on fused silica material for fiber optic sensors," in Proc. of SPIE, 2004, vol. 5342, pp. 128-36.

7. O. Leminger and R. Zengerle, "Determination of single-mode fiber coupler design parameters from loss measurements," IEEE J. of Lightwave Tech., vol. 3, pp. 864-867, 1985.

8. M. A. Jensen, R. H. Selfridge, "Analysis of etching-induced birefringence changes in elliptic core fibers," Applied Optics, vol. 31, pp. 2011-2016, 1992. 
9. S.M. Chandani, A. Kulpa, N.A.F. Jaeger, "Non-destructive determination of cladding thickness in D-fibers," IEEE Photonics Tech. Letters, vol. 18, pp. 1082-84, 2006.

10. A. Sharma, J. Kompella, P.K. Mishra, "Analysis of fiber directional couplers and coupler half-blocks using a new simple model for single-mode fibers," IEEE J. of Lightwave Tech., vol. 8, pp. 143-151, 1990.

11. D. J. Markos et al., "Controlled Core Removal from a D-Shaped Optical Fiber," Applied Optics, vol. 42, no. 36, pp. 7121-25, 2003.

12. Thomas D. Monte, KVH Industries, Inc., Tinley Park, IL, private communication, August 2006.

13. K. Thyagarajan, S. Diggavi, A.K. Ghatak, "Analytical investigations of leaky and absorbing planar structures," Optical and Quantum Electronics, vol. 19, pp. 131-137, 1987. 